\title{
Recent Progress on Correcting Components (useful for ELTs) at CILAS
}

\author{
J.-C. Sinquin ${ }^{1, a}$, J.-M. Lurçon ${ }^{1}$, C. Guillemard ${ }^{1}$ and P. Morin ${ }^{1}$ \\ ${ }^{1}$ CILAS, 8, avenue Buffon, Z.I. La Source, 45100 Orléans, France
}

Abstract. Recent progresses on correcting components at CILAS are exposed: the first one is relative to a $3.5 \mathrm{~mm}$ spacing, $28 \times 28$ order demonstrator for small pitch concave or convex deformable mirrors designed, manufactured and tested in the scope of feasibility study for the E-ELT MCAO module. The second one is relative to a $1 \mathrm{~mm}$ spacing, 50x50 order deformable mirror prototype. This prototyping allows the validation of some main important steps of manufacturing very high order deformable mirrors. This new piezo array family called "miniDM" uses transverse piezo-electrical effect. The last one is relative to a Tip/Tilt Mount prototype that has been designed and manufactured for the Narrow Field Infrared Adaptive Optics System (NFIRAOS), which is the MCAO system of the TMT. The system shows large angular stroke and bandwidth while supporting a heavy deformable mirror.

\section{Introduction}

Figure 1 shows an overview of piezo array DMs sorted according to stroke and spacing.

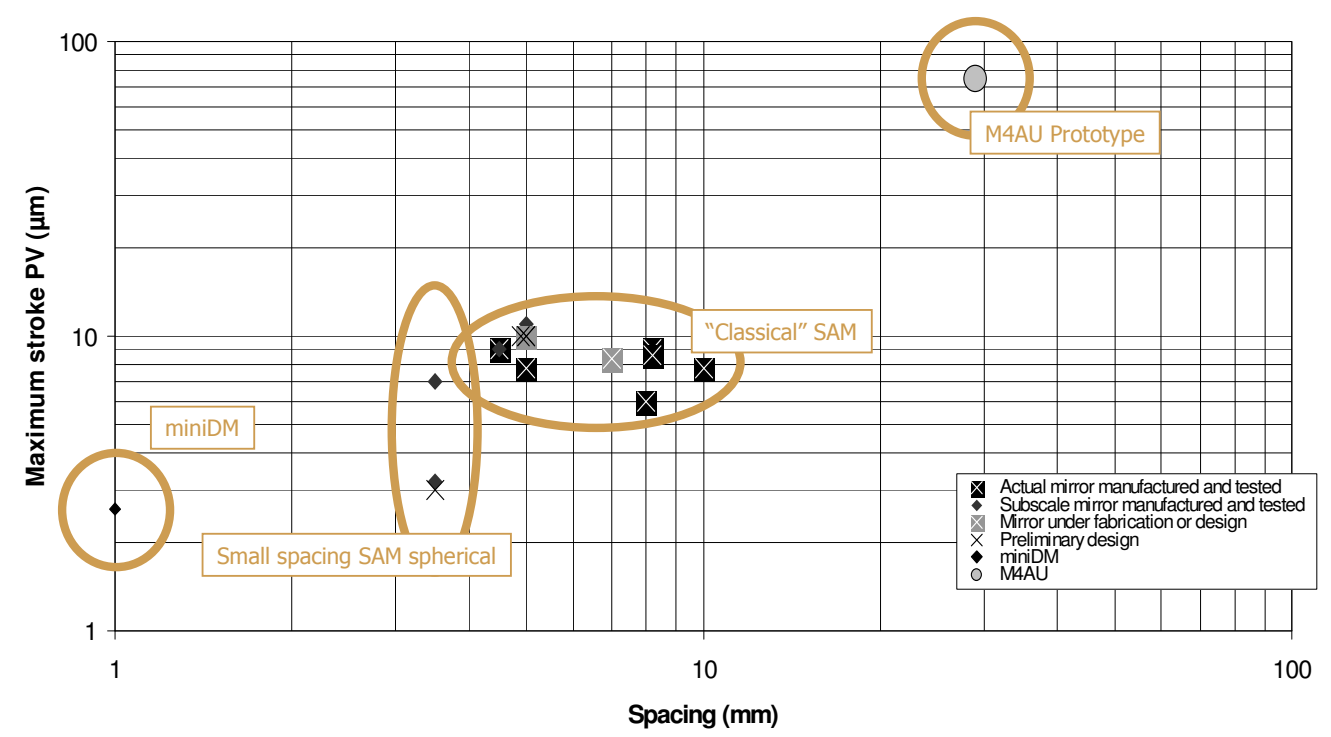

Figure 1. Different families of piezo array DMs

\footnotetext{
a e-mail: sinquin@cilas.com
} 
In the following, we will neither come back to "Classical" Stack Array Mirror (SAM) technology [1] nor to the specific technology developed for Adaptive Secondary Mirror (ASM) as M4AU prototype for E-ELT [2]. We will expose the experimental results obtained with "non classical" DM demonstrators that are $3.5 \mathrm{~mm}$ spacing spherical SAM and $1 \mathrm{~mm}$ spacing so called miniDM (both located on the left side of Figure 1).

Besides we will also expose the results obtained with a significantly new correcting component: the Tip/Tilt Mount prototype that shows large angular stroke and bandwidth while supporting a heavy DM.

\section{3.5-mm spacing Concave Deformable Mirror}

In the scope of feasibility study of two DMs for the E-ELT Multi Conjugate Adaptive Optics module showing the following main characteristics:

- high order (in the 100x100 range),

- small spacing $(<4 \mathrm{~mm})$,

- concave/convex surface (1-m range curvature radius),

- using lower voltage (200 V range),

- relatively low temperature operation $\left(-40^{\circ} \mathrm{C}\right)$,

we have designed, manufactured and tested a $3.5 \mathrm{~mm}$ spacing, $28 \times 28$ order demonstrator (see Figure 2). Its optical surface is $100 \mathrm{~mm}$ diameter and shows a $270 \mathrm{~mm}$ concave curvature radius.
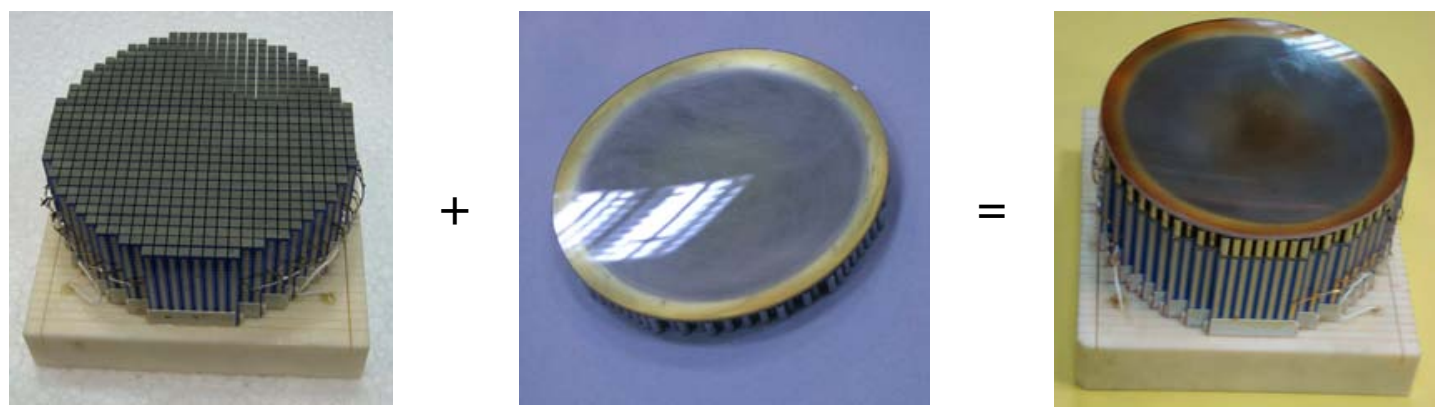

Figure 2. 28x28, 3.5-mm spacing concave DM demonstrator (from left to right: actuator array, curved optical head and assembled demonstrator)

With this demonstrator we obtained the following experimental results:

- Mechanical maximum stroke: $9.0 \mu \mathrm{m}$ PV for $\pm 400 \mathrm{~V}$

- Single stroke: $2.3 \mu \mathrm{m}$ PV

- Interactuator stroke: $1.5 \mu \mathrm{m}$

- Optical quality: high order WFE $<10 \mathrm{~nm}$ rms (calculated from shape at rest measurement)

- Temporal behaviour: resonance frequency $>20 \mathrm{kHz}$

- Non linearity (including hysteresis): $<5 \%$

- Thermal behaviour: strokes and optical quality have been also tested at $-40^{\circ} \mathrm{C}$; as temporal behaviour and non linearity they are equivalent to ambient.

The next step regarding this prototyping will see a reduction of the optical plate thickness in order to increase the local strokes up to:

- Single stroke: $3.8 \mu \mathrm{m}$ PV

- Interactuator stroke: $2.9 \mu \mathrm{m}$

while keeping the other parameters at the same level. 


\section{1-mm spacing Deformable Mirror (miniDM)}

In the scope of feasibility demonstration of DM showing:

- high density (in the 200x200 range),

- small spacing (1 mm range),

which is one of the key elements of the future Extreme Adaptive Optics systems for the E-ELT, with the support of ESO and LAOG, we have developed a $1 \mathrm{~mm}$ spacing deformable mirror prototype with 50x50 actuators (see Figure 3). This prototyping is aimed at the validation of some main important steps of manufacturing very high order deformable mirrors. These steps are machining and connecting processes. This new piezo array family called "miniDM" uses transverse piezo-electrical effect, which fits well the small spacing requirement, but shows architecture and materials very close to classical SAM, which is decisive regarding general behaviour and environmental compatibility.
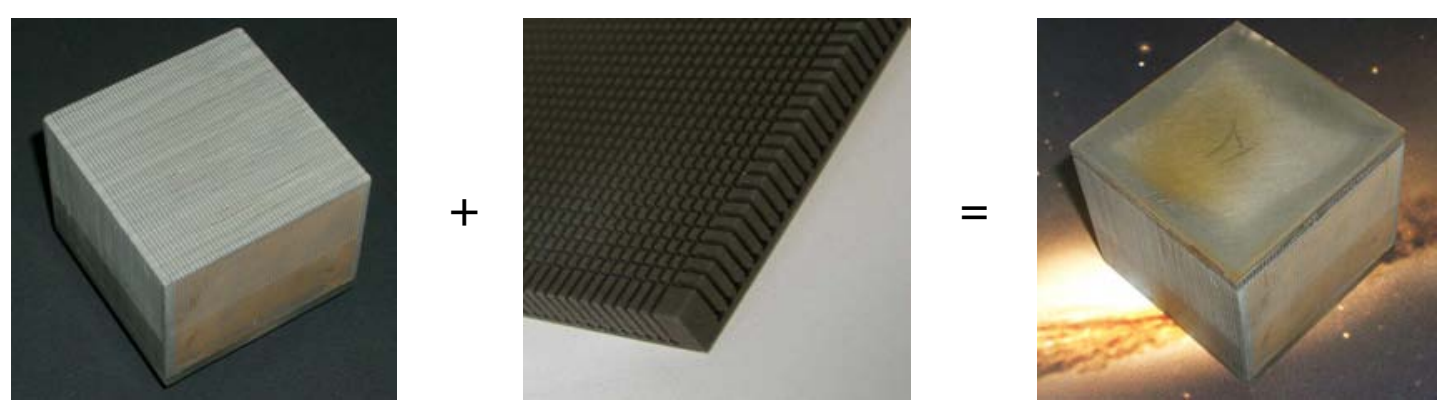

Figure 3. 50x50, 1-mm spacing DM prototype - miniDM

(from left to right: actuator array, back side of optical head and assembled prototype)

With this prototype we obtained the following experimental results:

- Mechanical maximum stroke: $2.9 \mu \mathrm{m}$ PV for $\pm 400 \mathrm{~V}$

- Single stroke: $0.8 \mu \mathrm{m}$ PV

- Interactuator stroke: $0.5 \mu \mathrm{m}$

- Optical quality: high order WFE $<6 \mathrm{~nm}$ rms (calculated from shape at rest measurement)

- Temporal behaviour: resonance frequency $>30 \mathrm{kHz}$

- Non linearity (including hysteresis): $6 \%$

These results show that we demonstrated the use of the transverse effect of piezo material to design an array DM. We also succeeded to manufacture a 50x50 1-mm spacing array and the associated optical head and we assembled them together. Nevertheless two aspects are not demonstrated yet:

- The electrical contacting and insulation processes we used are neither industrial nor reliable; therefore some complementary studies and manufacturing of simple breadboards need to be done (some promising tracks are available).

- The obtained surface cosmetics is poor and must be improved by some additional works on the optical head.

These improvements will be done during the next development phase. 


\section{Tip/Tilt Mount for heavy SAM}

A Tip/Tilt Mount prototype has been designed and manufactured for the Narrow Field Infrared Adaptive Optics System (NFIRAOS), which is the MCAO system of the TMT. This Tip/Tilt Mount, which comes from Observatoire de Paris concepts, will be used to correct tip/tilt jitter disturbances. These disturbances include atmospheric turbulence and telescope vibration effects caused by wind shake and mechanical vibration.

The design of the Tip/Tilt Mount (see Figure 4) consists in building a mechanical gimbals mount around the DM. The central ring holds and supports the DM assembly with a dedicated interface. Thanks to the intermediate ring, the gimbals mount provides two perpendicular rotation axes. A pair of specific stiff flexible pivots for each axis defines the rotation axis position without any friction. The tilt rotation axis is located at the centre of gravity of the moving part which is very close of the centre of gravity of the DM. This allows the lowest power dissipation. Two linear actuators (voice coil type) and two position sensors (capacitive type) are implemented on each axis for the position servo loop control. The system is held by a specific rigid chassis.

At the present step, the SAM is replaced by a dummy DM showing the same mechanical properties as the future mirror (for instance its mass is $32 \mathrm{~kg}$ ). The $3125 \mathrm{high}$ voltage wires are also connected between the dummy DM and the back side of the chassis.
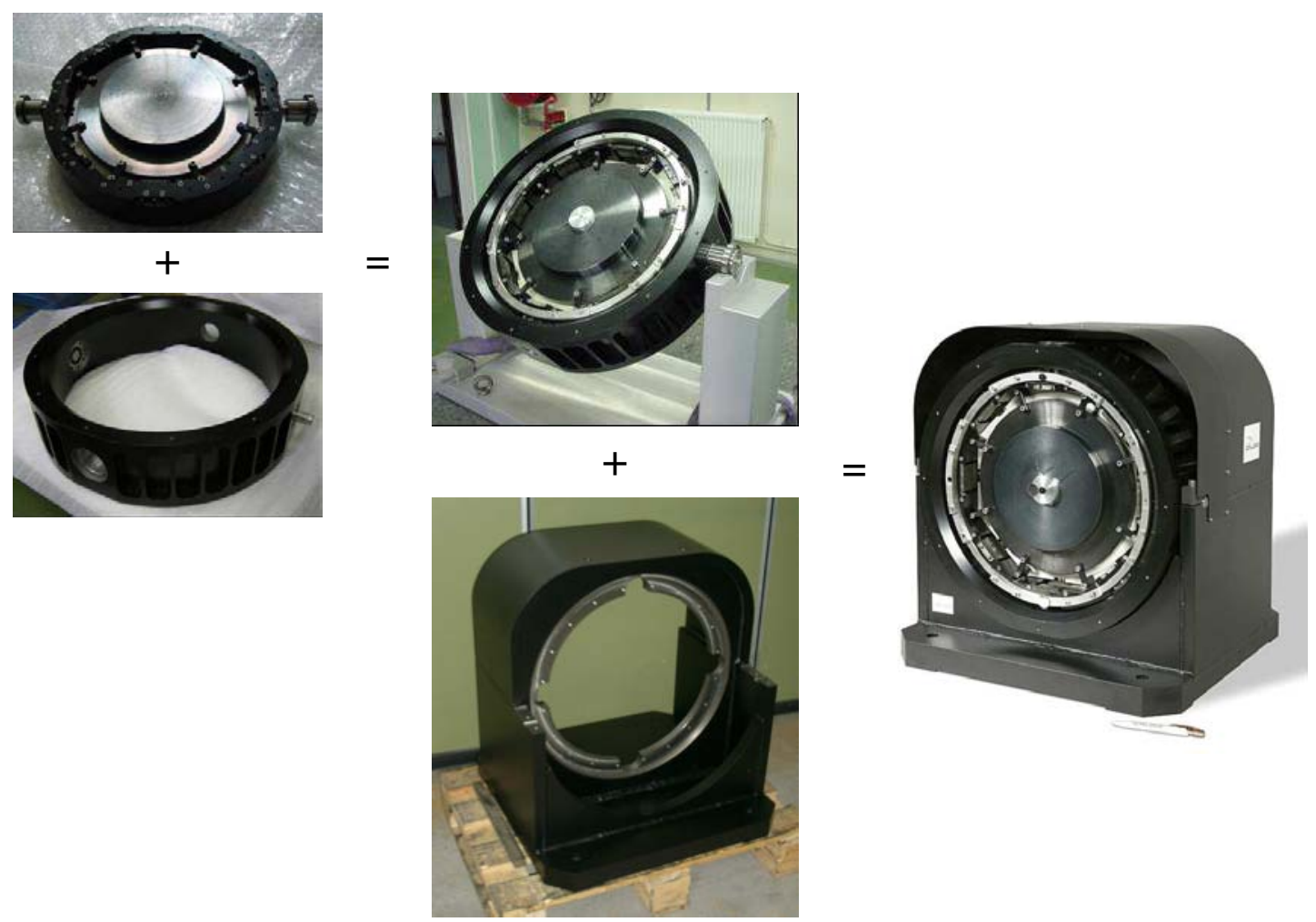

Figure 4. Tip/Tilt Mount prototype

(from left to right: central ring with dummy DM, intermediate ring, equipped gimbals, chassis and overall assembly) 
The system was first tested in open loop and showed its vertical and horizontal tilt natural resonance frequencies at 11 and $19 \mathrm{~Hz}$ close to theoretically expected. Then the closed loop behaviour was tested at ambient and at $-35^{\circ} \mathrm{C}$; the experimental results are the same at both temperatures, they are given hereafter:

- Low frequency angular stroke: $500 \mu \mathrm{rad}$ PV

- Angular stroke at $20 \mathrm{~Hz}:>25 \mu \mathrm{rad}$ PV

- Achievable closed-loop, small amplitude -3dB bandwidth: $>90 \mathrm{~Hz}$

- Angular resolution and noise: $<40$ nrad rms

- Linearity and hysteresis: limited by phase lag

Note that the significantly high bandwidth of the system has been obtained using a numerical corrector with a dumping loop on speed and a Predictive Functional Control (PFC) on position.

The next step will be an upgrade of the system in order to provide the level of reliability, maintainability, and ease of integration/use appropriate for NFIRAOS, while still preserving the basic functionality and tip/tilt correction performance already demonstrated by the prototype.

\section{Acknowledgements}

A significant part of presented developments has been supported by ESO, LAOG and OPTICON, TMT, NRC/HIA and University of Toronto; we would like to thank them here.

\section{References}

1. J.-C. Sinquin, J.-M. Lurçon, C. Guillemard, Proceedings of SPIE 7015, 23 (2008)

2. B. Crepy et al., "Piezostack technology for the M4 adaptive unit of the E-ELT," Presented at the 1st AO4ELT conference (Paris, June 2009) 